\title{
The delay time distribution of massive double compact star mergers (Research Note)
}

\begin{abstract}
N. Mennekens and D. Vanbeveren
Astronomy and Astrophysics Research Group, Vrije Universiteit Brussel, Pleinlaan 2, 1050 Brussels, Belgium e-mail: [nmenneke;dvbevere]@vub.ac.be

Received 26 January 2016 / Accepted 8 March 2016

ABSTRACT

To investigate the temporal evolution of binary populations, in general, and double compact-star binaries and mergers, in particular, within a galactic evolution context, a very straightforward method is obviously to implement a detailed binary evolutionary model in a galactic chemical evolution code. To our knowledge, the Brussels galactic chemical evolution code is the only one that fully and consistently accounts for the important effects of interacting binaries on the predictions of chemical evolution. With a galactic code that does not explicitly include binaries, the temporal evolution of the population of double compact star binaries and mergers can be estimated with reasonable accuracy if the delay time distribution (DTD) for these mergers is available. The DTD for supernovae type Ia has been studied extensively in the past decade. In the present paper we present the DTD for merging double neutron-star binaries and mixed systems consisting of a neutron star and a black hole. The latter mergers are very promising sites for producing $r$-process elements, and the DTDs can be used to study the galactic evolution of these elements with a code that does not explicitly account for binaries.
\end{abstract}

Key words. binaries: close - stars: massive - Galaxy: evolution

\section{Introduction}

The $r$-process nucleosynthesis has been known since the pioneering works of Burbidge et al. (1957) and Seeger et al. (1965), but the main production site of the $r$-process elements still remains a matter of debate. Basically, two sites have been studied in some detail in the recent past: type-II supernova ( $\mathrm{SN}$; including long gamma-ray bursts) and merging double compact-star binaries, either double neutron star systems (NS+NS) or neutron star + black hole binaries (NS+BH). Type-II SN may have been main $r$-process sites in the early-time Galaxy when $[\mathrm{Fe} / \mathrm{H}]<$ -3 , but it is questionable that they remained important when the metallicity increased (e.g. Qian 2012; Wanajo et al. 2011; Janka 2012). Recent hydrodynamical simulations of the merging process of double compact-star binaries have shown that during and after the merger phase some $10^{-3} M_{\odot}$ up to more than $0.1 M_{\odot}$ of neutron-rich matter can be ejected from the system (e.g. Rosswog 2005; Oechslin et al. 2007; Goriely et al. 2011; Korobkin et al. 2012; Bauswein et al. 2013; Wanajo et al. 2014; Foucart et al. 2014; Just et al. 2015). Although the results of the above-mentioned simulations may be quantitatively different, they all agree that the neutron-rich ejecta provide the necessary conditions for very efficient $r$-processing.

By implementing the details of one particular $r$-process site in a chemical evolutionary code, it is possible to compute the galactic temporal variation of $r$-process elements predicted by that site. Comparison with observations (mainly the observations of Eu) may yield important information about the importance of the chosen site for the overall galactic $r$-process production. In principle, if one decides to study the effects of merging double compact-star binaries in this way, for consistency, one has to combine a full binary population model (including binary chemical yields) with a galactic formation and evolution code. We would like to remind the interested reader that present observations of massive stars yield a massive interacting binary frequency of at least 70\% (Sana et al. 2012), and we note that most of the existing chemical evolution codes do not account for the evolutionary consequences of binaries. Our code, described in De Donder \& Vanbeveren (2004) with important updates listed in Vanbeveren et al. (2012) and in Mennekens \& Vanbeveren (2014), is to our knowledge the only code with an internal and fully self-consistent detailed binary population model (including binary yields). We used this code to predict the temporal evolution of the $r$-process elements ejected by double compact-star binaries. An important conclusion resulting from our 2004 and 2014 results was that (with exception for the first, say, $100 \mathrm{Myr}$ ) compact-star binary mergers may be major contributors to the $r$-process enrichment and that it is very plausible that this enrichment is mainly due to $\mathrm{BH}+\mathrm{NS}$ mergers.

Matteucci et al. (2014) tried to evaluate the importance of double neutron-star mergers as an $r$-process production site; however, since they used a galactic code that does not explicitly account for binaries, approximations had to be made that may affect overall conclusions. To illustrate, the authors adopt a model with three fixed merger delay timescales for all double compact-star mergers, and they correctly note that a more realistic approach would consider a distribution function on such timescales. Moreover, since their code does not account for close binaries, they have to rely on "observed" merger rates like those proposed by Kalogera et al. (2004). However, these rates may tell us something about the present situation, but they do not give information on the temporal (Z-dependent) evolution of the 
merger rates. Moreover, the rates are based on the observation of only 9-10 observed double NS binaries and are therefore very uncertain. Last but not least, no observations exist on BH+NS binaries, and as already discussed above, merging BH+NS may be the dominant $r$-process production site.

Scientists that use chemo-dynamical models to study galactic evolution rarely include binaries, and they are a requesting party for time delay distributions of merging double compactstar binaries.

We find ourselves in a privileged position for providing these distributions, and this is the main goal of the present paper. It should not be considered as a stand-alone paper but rather as an addendum to Mennekens \& Vanbeveren (2014, Paper I).

\section{The delay time distribution (DTD) of double compact-star mergers}

The delay time is defined as the evolutionary time $\left(\Delta t_{\text {evol }}\right)$ between the formation of a massive binary and the eventual formation of the double compact star system $(\mathrm{NS}+\mathrm{NS}$ or $\mathrm{NS}+\mathrm{BH})+$ the coalescence timescale $\left(\Delta t_{\mathrm{mer}}\right)$ of the latter because of relativistic gravitational wave emission. In our simulations, $\Delta t_{\mathrm{evol}}$ is computed from first binary evolution principles. In binaries where RLOF and mass transfer is active, this evolutionary time is not simply the evolutionary time of the low-mass companion of the binary. The coalescence time obviously depends on the period of the double compact-star binary, but it is also very dependent on its eccentricity. To calculate the DTD, it is therefore indispensable to calculate the effects of an SN explosion in detail (which may be asymmetric) on the binary orbital parameters. Our binary code has been introduced in De Donder \& Vanbeveren (2004), and important updates were described in Vanbeveren et al. (2012) and Mennekens \& Vanbeveren (2014). We invite the interested reader to consider at least the last since the present paper is written as an addendum to it.

To summarise these papers, our binary population code accounts for the following binary processes: (i) stable RLOF in Case $\mathrm{A}$ and $\mathrm{Br}^{1}$ binaries, accounting for conservative and nonconservative assumptions characterised by the mass transfer efficiency $\beta$; (ii) common-envelope evolution in Case $\mathrm{Bc}$ and $\mathrm{C}$ binaries, characterised by the energy conversion efficiency $\alpha$; (iii) common-envelope evolution of a binary where one of the components is a compact star; (iv) a detailed treatment of the effects of the supernova explosion on binary parameters (the SN can be asymmetric, and the degree of asymmetry is expressed as the kick velocity that the compact star gets, which also depends on the adopted fallback mechanism as described in Fryer et al. 2012); (v) stars that end their life in a prompt electron capture supernova (ECSN) where the resulting neutron star is formed with a small kick (Podsiadlowski et al. 2004); (vi) and finally the formation of a BH with or without a SN explosion. Another important assumption is whether or not a luminous blue variable (LBV) phase can strip the entire envelope of a very massive ( $>40 M_{\odot}$ ) star in a spherically symmetric way. It is obvious that the code also accounts for the details of stellar evolution, which depends on e.g. stellar wind mass loss, convective core overshooting, mass accretion during stable RLOF in case $\mathrm{A} / \mathrm{Br}$ binaries, and the binary merger process.

Once a double compact-star binary is formed (i.e., after the second SN explosion of the binary) our code computes

\footnotetext{
1 We follow the Cases A, Br, Bc, and C binary classification of Kippenhahn \& Weigert (1967) and Lauterborn (1970).
}

the coalescence timescale using the gravitational wave radiation formalism of Landau \& Lifshitz (1951). The formalism is summarised in De Donder \& Vanbeveren (2004). We repeat that the timescale strongly depends on the eccentricity of the binary, and therefore a detailed model that computes the effects of an asymmetric SN explosion on binary parameters is absolutely necessary.

A DTD depends on the various distribution functions of star and binary parameters. In correspondence with current observations of massive stars and binaries in our Galaxy, we adopt (De Donder \& Vanbeveren 2004) (i) a Scalo (1986) initial mass function $\sim M^{-2.7}$ for single stars and for primaries of close binaries with mass $>2 M_{\odot}$ and a Salpeter (1955) slope $\sim M^{-2.35}$ for $0.08 \leq M / M_{\odot} \leq 2$; (ii) a flat binary mass ratio distribution and (iii) a binary period distribution that is flat in the Log. Recent observations of Sana et al. (2012) support the flat mass ratio distribution, however the authors conclude that a period distribution $\sim(\log \mathrm{P})^{-0.55}$ is possible. In Paper I we investigated the effect on the population of double compact-star systems and concluded that it is marginal. To support this conclusion we also compute the DTD with the "alternative" period distribution.

The effects of an asymmetric SN explosion on binary parameters are computed by relating the asymmetry and the kick the compact star gets. We therefore need a distribution function of possible kicks. The kick velocity distribution is linked to the observed pulsar velocity distribution, and we use the study of Lyne \& Lorimer (1994). This yields a $\chi^{2}$-distribution with average $450 \mathrm{~km} \mathrm{~s}^{-1}$, but to account for possible observational errors, we also calculated the DTD using a distribution with average $265 \mathrm{~km} \mathrm{~s}^{-1}$ (Hobbs et al. 2005).

\section{Results}

The binary population models for which a DTD of double compact star mergers have been calculated are those described in Paper I and listed in Table 1 of that paper. Since the latter concerned galactic evolution, Paper I also considered various star formation rate models (which is, for example, the only difference between Models 2 and 4), which is obviously not important here. Furthermore, only those models are withdrawn that predict a current Galactic double neutron star merger rate ${ }^{2} \geq 3 / \mathrm{Myr}$, which is a most probable observational lower limit (Kim et al. 2010). According to the results of Table 2 in Paper I, Models 1 , $3,8,9,10,11,17,18,22$ do not meet this lower limit. Model 22 predicts a merger rate of $2.5 / \mathrm{Myr}$, which means that rejecting this model is, at first glance, not based on a strong argument. An additional argument for rejecting Model 22 comes from the predicted DTD of Type Ia supernovae if the same binary parameter values are used as in Model 22. The SN Ia DTD predicted by the Brussels code has been described in detail in Mennekens et al. (2010) and is shown in Fig. 1 if the model 22 parameters are used. As can be noticed, the theoretically predicted DTD deviates from observations by a large factor, and this strengthens the conclusion to reject Model 22.

Using our Galactic chemical evolution code that includes a detailed treatment of binaries, we also computed the predicted temporal evolution of the $r$-process elements for all the binary models. It is clear that the models that predict too much $r$-process element enrichment have to be rejected as well ${ }^{3}$. This is the case

\footnotetext{
2 This is the same rate as calculated by e.g. Dominik et al. (2012).

3 Any $r$-process element production process that has not yet been recognised and/or has not yet been included in our simulations will obviously strengthen this conclusion.
} 


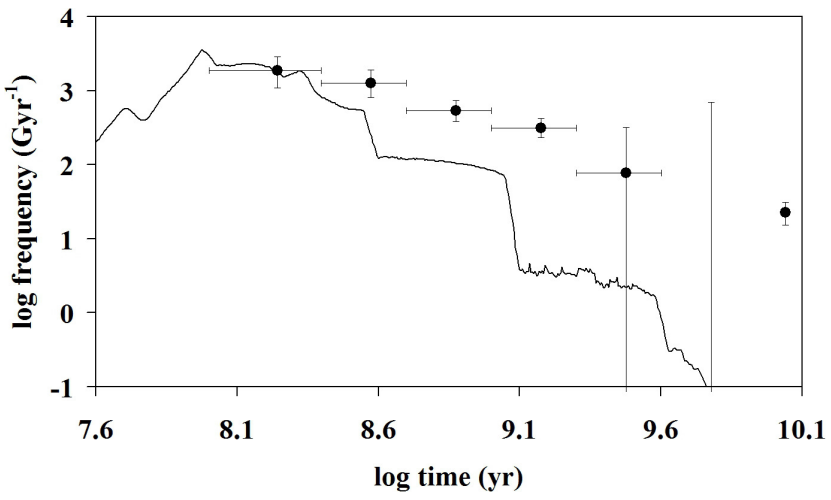

Fig. 1. SN Ia DTD obtained using Model 22 from Paper I (solid line) compared to observations (Totani et al. 2008; dots). See Mennekens et al. (2010) for details on the computation and comparison methods.

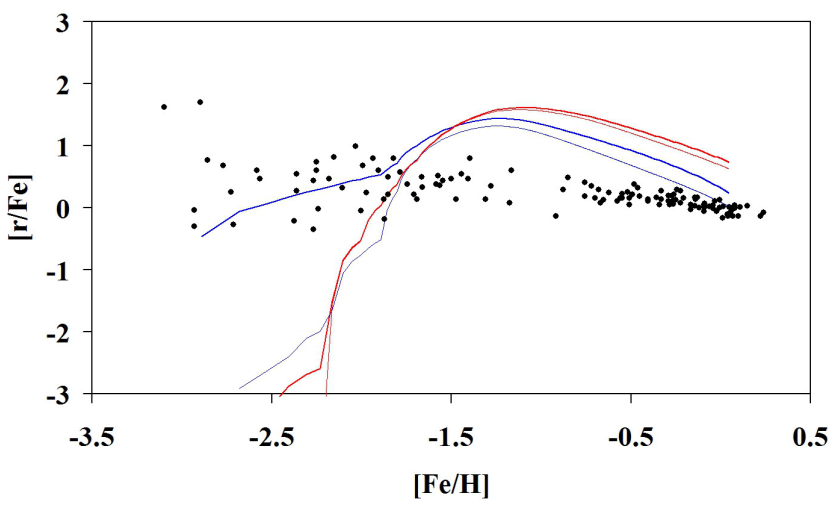

Fig. 2. $r$-process element enrichment obtained using Models 6 (blue) and 19 (red) from Paper I. The thin lines indicate enrichment taking only NSNS mergers into account. The black dots represent observations (see Mennekens \& Vanbeveren 2014).

for Models 6, 14, 15, 16, 19, 20, 21, 23. To illustrate this criterion Fig. 2 shows the predicted temporal evolution of $r$-process enrichment for two representative binary models, and we compare with observations ${ }^{4}$. Interestingly, the models that do not predict too much $r$-process enrichment currently have a combined galactic NS+NS and NS+BH merger rate $\leq 20 / \mathrm{Myr}(\leq 10 / \mathrm{Myr}$ for both).

Tables A.1 and A.2 give the DTD of NS+NS and of $\mathrm{NS}+\mathrm{BH}$ mergers for the non-rejected models as discussed above. Table A.5 reminds the reader of the assumptions behind these models, which are laid out in more detail in Paper I. The calculated rates are for a starburst with total mass $=1 M_{\odot}$, and a binary frequency $=100 \%$. It is then obvious to estimate the rates for any starburst regardless of the binary frequency. In Tables A.3 and A.4, we give the corresponding DTDs of the $r$-process yields by linking the calculations of Korobkin et al. (2012) with our predicted temporal evolution of the population of compact star mergers. Figure 3 shows the DTD for Model 2.

All the models retained in the present paper give very similar DTDs. The DTDs illustrate that NS+NS or/and NS+BH mergers show up typically 10-30 Myr after the onset of the starburst, whereas the merger rates depend critically on the metallicity $Z$. Figure 5 is similar to Fig. 3 but for the binary period distribution proposed by Sana et al. (2012). As can be noticed, the difference is moderate.

4 We use the same observations as those discussed in Paper I.

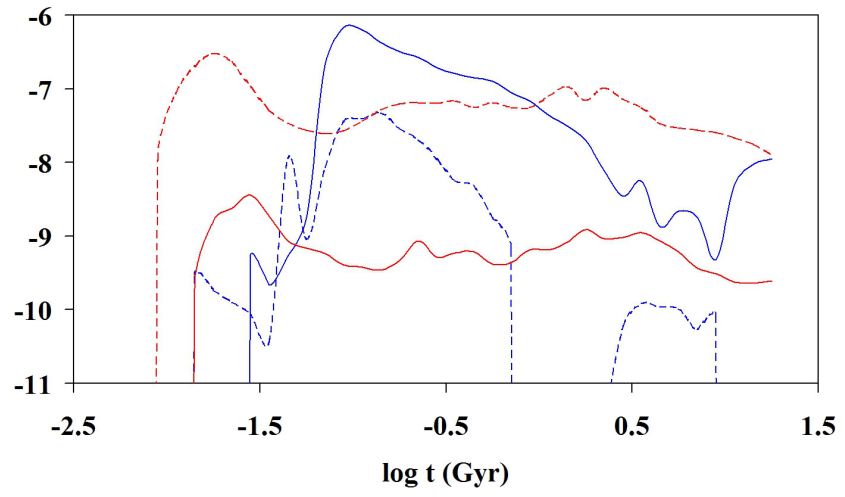

Fig. 3. DTD of double compact-star mergers obtained using Models 2 or 4 , in logarithmic number of events per time bin after a starburst with total mass $1 M_{\odot}$. Blue lines indicate NSNS mergers, red lines BHNS mergers. Dashed lines indicate $Z=0.002$, solid lines $Z=0.02$.

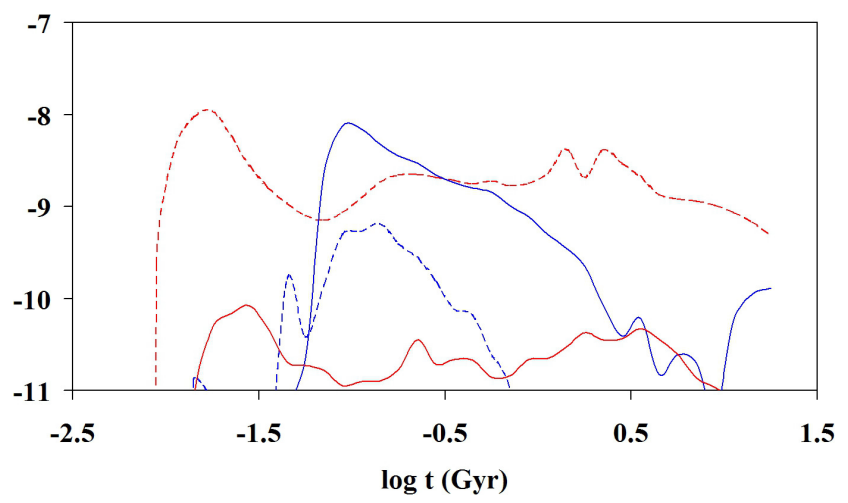

Fig. 4. DTD of double compact-star-merger $r$-process element yields obtained using Models 2 or 4 , in logarithmic $M_{\odot}$ per time bin after a starburst with total mass $1 M_{\odot}$. Blue lines indicate NSNS mergers, red lines BHNS mergers. Dashed lines indicate $Z=0.002$, solid lines $Z=0.02$.

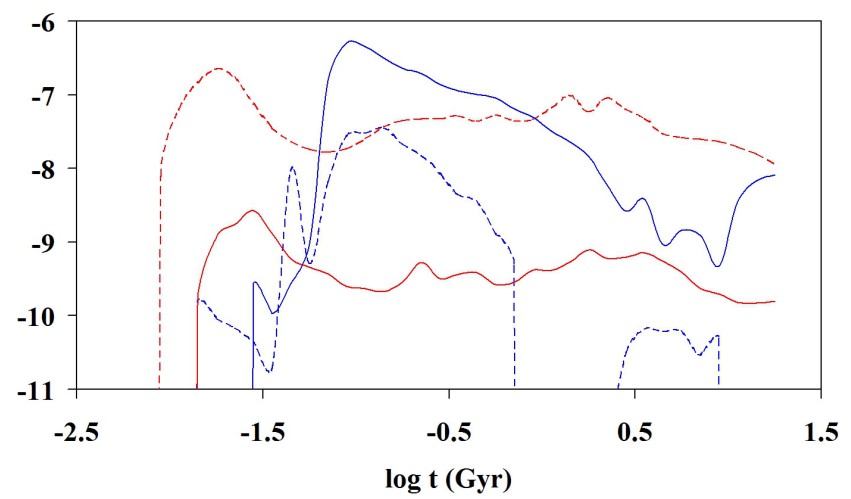

Fig. 5. DTD of double compact-star mergers obtained using Model 13 in logarithmic number of events per time bin after a starburst with total mass $1 M_{\odot}$. Blue lines indicate NSNS mergers, red lines BHNS mergers. Dashed lines indicate $Z=0.002$, solid lines $Z=0.02$.

\section{Comparison to earlier work}

Many research groups studied the populations of massive compact star pairs, and some of them discuss the DTDs. One of the earliest papers is the one of Tutukov \& Yungelson (1994), a paper published in the era when it was believed that the supernova explosion of a massive star is spherically symmetric. The results of Tutukov \& Yungelson were calculated with the 
latter assumption. For more recent work (with in particular a non-spherical treatment of the SN explosion), we would like to refer to De Donder \& Vanbeveren (2004) and Dominik et al. (2012) and references therein. In all these studies, the delay time is defined as the orbital decay time between the formation of the double compact-star binary and the moment of merging, and obviously the published distributions describe the distributions of these decay times. However, this is not the one needed for studying the effect of massive binaries on the $r$-process element enrichment of galaxies with a code that does not explicitly account for binaries. What is really needed is the DTD starting from a binary population on the zero-age main sequence up to the double compact-star merger, i.e. the delay time must include the evolutionary timescale of the progenitor binary. The DTDs presented here account for this, and therefore they can hardly be compared to the DTDs presented in earlier work.

\section{Conclusion}

The theoretically predicted DTD of NS+NS and of NS+BH mergers depends on binary parameters whose values are poorly constrained from first principles. However, a careful comparison with observations allows promoting a very plausible DTD that can be easily implemented in a galactic chemical code in this way, thereby avoiding the need to add a full binary population model. This may, in particular, be very useful for studying the $r$-process element enrichment of dwarf galaxies, which are the building blocks of spirals. Our results reveal that the galactic chemo-binary models which predict an $r$-process element enrichment not too large compared to observations all have a current galactic double neutron-star merger rate $\leq 10 / \mathrm{Myr}$ (corresponding to a combined $\mathrm{NS}+\mathrm{NS}$ and $\mathrm{NS}+\mathrm{BH}$ merger rate $\leq 20 / \mathrm{Myr}$ ). The latter rate obviously has very important consequences for the analysis and interpretation of the results of aLIGO, which will become available in the very near future.
Acknowledgements. We thank the anonymous referee for valuable comments.

\section{References}

Bauswein, A., Goriely, S., \& Janka, H.-T. 2013, ApJ, 773, 78 Burbidge, E., Burbidge, G., Fowler, W., \& Hoyle F. 1957, Rev. Mod. Phys., 29, 547

De Donder, E., \& Vanbeveren, D. 2004, New Astron. Rev., 48, 861

Dominik, M., Belczynski, K., Fryer, C., et al. 2012, ApJ, 759, 52

Foucart, F., Deaton, M. B., Duez, M. D., et al. 2014, Phys. Rev. D, 90, 024026

Fryer, C., Belczynski, K., Wiktorowicz, G., et al. 2012, ApJ, 749, 91

Goriely, S., Bauswein, A., \& Janka, H.-T. 2011, ApJ, 738, L32

Hobbs, G., Lorimer, D., Lyne, A., \& Kramer, M. 2005, MNRAS, 360, 974

Janka, H.-T. 2012, Ann. Rev. Nucl. Part. Sci., 62, 407

Just, O., Bauswein, A., Ardevol Pulpillo, R., et al. 2015, MNRAS, 448, 541

Kalogera, V., Kim, C., Lorimer, D. R., et al. 2004, ApJ, 601, L179

Kim, C., Kalogera, V., \& Lorimer, D. 2010, New Astron. Rev., 54, 148

Kippenhahn, R., \& Weigert, A. 1967, Z. Astrophys., 65, 251

Korobkin, O., Rosswog, S., Arcones, A., \& Winteler, C. 2012, MNRAS, 426, 1940

Landau, L., \& Lifshitz, E. 1951, in The Classical Theory of Fields (Pergamon Press)

Lauterborn, D. 1970, A\&A, 7, 150

Lyne, A., \& Lorimer, D. 1994, Nature, 369, 127

Matteucci, F., Romano, D., Arcones, A., et al. 2014, MNRAS, 438, 2177

Mennekens, N., \& Vanbeveren, D. 2014, A\&A, 564, A134 (Paper I)

Mennekens, N., Vanbeveren, D., De Greve, J.-P., \& De Donder, E. 2010, A\&A, 515, A89

Oechslin, R., Janka, H., \& Marek, A. 2007, A\&A, 467, 395

Podsiadlowski, P., Langer, N., Poelarends, A., et al. 2004, ApJ, 612, 1044

Qian, Y.-Z. 2012, AIP Conf. Proc., 1484, 201

Rosswog, S. 2005, ApJ, 634, 1202

Salpeter, E. 1955, ApJ, 121, 161

Sana, H., De Mink, S., De Koter, A., et al. 2012, Science, 337, 444

Scalo, J. 1986, Fund. Cosmic Phys., 11, 1

Seeger, P., Fowler, W., \& Clayton D. 1965, ApJS, 11, 121

Totani, T., Morokuma, T., Oda, T., et al. 2008, PASJ, 60, 1327

Tutukov, A., \& Yungelson, L. 1994, MNRAS, 268, 871

Vanbeveren, D., Mennekens, N., \& De Greve, J.-P. 2012, A\&A, 543, A4

Wanajo, S., Janka, H.-T., \& Müller, B. 2011, ApJ, 726, L15

Wanajo, S., Sekiguchi, Y., Nishimura, N., et al. 2014, ApJ, 789, L39 


\section{Appendix A: Tables}

Table A.1. Double compact-star merger DTD for $Z=0.002$.

\begin{tabular}{|c|c|c|c|c|c|c|c|c|c|c|}
\hline $\begin{array}{l}\log t \\
(\mathrm{Gyr})\end{array}$ & $\begin{array}{l}\text { mod. } 2 / 4 \\
\text { NSNS }\end{array}$ & $\begin{array}{c}\text { mod. 2/4 } \\
\text { BHNS }\end{array}$ & $\begin{array}{l}\text { mod. } 5 \\
\text { NSNS }\end{array}$ & $\begin{array}{l}\text { mod. } 5 \\
\text { BHNS }\end{array}$ & $\begin{array}{l}\text { mod. } 7 \\
\text { NSNS }\end{array}$ & $\begin{array}{l}\text { mod. } 7 \\
\text { BHNS }\end{array}$ & $\begin{array}{c}\text { mod. } 12 \\
\text { NSNS }\end{array}$ & $\begin{array}{c}\text { mod. } 12 \\
\text { BHNS }\end{array}$ & $\begin{array}{l}\text { mod. } 13 \\
\text { NSNS }\end{array}$ & $\begin{array}{c}\text { mod. } 13 \\
\text { BHNS }\end{array}$ \\
\hline-2.05 & & -7.973 & & -7.848 & & -7.360 & & & & -8.116 \\
\hline-1.95 & & -7.051 & & -7.005 & & -7.370 & & & & -7.190 \\
\hline-1.85 & -9.521 & -6.699 & -8.981 & -6.632 & -9.449 & -7.163 & -9.521 & -9.229 & -9.816 & -6.836 \\
\hline-1.75 & -9.735 & -6.515 & -9.932 & -6.421 & -9.524 & -7.230 & -9.735 & -10.340 & -10.035 & -6.650 \\
\hline-1.65 & -9.897 & -6.639 & -10.398 & -6.610 & -9.277 & -6.872 & -9.897 & -9.849 & -10.165 & -6.771 \\
\hline-1.55 & -10.065 & -6.976 & -9.085 & -6.958 & -8.315 & -6.855 & -10.065 & -8.902 & -10.349 & -7.121 \\
\hline-1.45 & -10.438 & -7.288 & -9.565 & -7.271 & -7.738 & -6.786 & -10.438 & -9.132 & -10.714 & -7.451 \\
\hline-1.35 & -7.943 & -7.460 & -7.899 & -7.427 & -7.418 & -6.911 & -7.943 & -9.211 & -8.012 & -7.636 \\
\hline-1.25 & -9.040 & -7.564 & -9.049 & -7.528 & -7.450 & -6.745 & -9.040 & -9.331 & -9.294 & -7.743 \\
\hline-1.15 & -8.072 & -7.608 & -8.067 & -7.560 & -6.455 & -6.681 & -8.072 & -9.457 & -8.185 & -7.783 \\
\hline-1.05 & -7.451 & -7.566 & -7.422 & -7.502 & -6.300 & -6.724 & -7.451 & -9.915 & -7.561 & -7.732 \\
\hline-0.95 & -7.408 & -7.441 & -7.389 & -7.367 & -6.515 & -6.731 & -7.408 & -9.793 & -7.525 & -7.595 \\
\hline-0.85 & -7.328 & -7.287 & -7.311 & -7.213 & -6.817 & -6.791 & -7.328 & -9.702 & -7.452 & -7.436 \\
\hline-0.75 & -7.536 & -7.209 & -7.425 & -7.139 & -6.981 & -6.794 & -7.536 & -9.933 & -7.667 & -7.351 \\
\hline-0.65 & -7.692 & -7.187 & -7.677 & -7.127 & -7.161 & -6.816 & -7.692 & -10.494 & -7.824 & -7.324 \\
\hline-0.55 & -7.961 & -7.198 & -8.045 & -7.141 & -7.285 & -6.812 & -7.961 & -10.577 & -8.073 & -7.327 \\
\hline-0.45 & -8.250 & -7.166 & -8.177 & -7.168 & -7.504 & -6.787 & -8.250 & -11.083 & -8.350 & -7.287 \\
\hline-0.35 & -8.325 & -7.251 & -9.003 & -7.196 & -7.730 & -6.763 & -8.325 & -9.968 & -8.452 & -7.363 \\
\hline-0.25 & -8.758 & -7.187 & -10.298 & -7.217 & -7.932 & -6.688 & -8.758 & -7.546 & -8.888 & -7.280 \\
\hline-0.15 & -9.181 & -7.250 & & -7.231 & -8.236 & -6.676 & -9.181 & -9.010 & -9.319 & -7.349 \\
\hline-0.05 & & -7.251 & & -7.239 & -8.540 & -6.601 & & -8.005 & & -7.332 \\
\hline 0.05 & & -7.103 & & -7.234 & -8.767 & -6.588 & & -8.405 & & -7.171 \\
\hline 0.15 & & -6.971 & & -7.227 & -9.308 & -6.489 & & -8.023 & & -7.013 \\
\hline 0.25 & & -7.155 & & -7.213 & -9.426 & -6.436 & & -7.461 & & -7.230 \\
\hline 0.35 & -12.049 & -6.988 & & -7.206 & -9.751 & -6.562 & -12.049 & -7.537 & -12.333 & -7.043 \\
\hline 0.45 & -10.227 & -7.140 & -10.249 & -7.205 & -9.281 & -6.477 & -10.227 & -7.535 & -10.496 & -7.198 \\
\hline 0.55 & -9.915 & -7.255 & -9.579 & -7.202 & -8.896 & -6.432 & -9.915 & -7.294 & -10.181 & -7.318 \\
\hline 0.65 & -9.966 & -7.474 & -9.484 & -7.190 & -8.414 & -6.582 & -9.966 & -7.524 & -10.214 & -7.529 \\
\hline 0.75 & -9.988 & -7.529 & -9.672 & -7.202 & -7.811 & -6.503 & -9.988 & -7.438 & -10.212 & -7.577 \\
\hline 0.85 & -10.274 & -7.559 & -9.988 & -7.226 & -7.637 & -6.616 & -10.274 & -7.584 & -10.542 & -7.604 \\
\hline 0.95 & -10.074 & -7.593 & -9.918 & -7.266 & -7.601 & -6.573 & -10.074 & -7.547 & -10.308 & -7.636 \\
\hline 1.05 & & -7.665 & -10.297 & -7.326 & -7.592 & -6.707 & & -7.520 & & -7.710 \\
\hline 1.15 & & -7.758 & -10.896 & -7.378 & -7.555 & -6.717 & & -7.408 & & -7.804 \\
\hline 1.25 & & -7.895 & -11.535 & -7.453 & -7.628 & -6.666 & & -7.475 & & -7.940 \\
\hline
\end{tabular}

Notes. Values are logarithmic, in number of events per time bin after a starburst with total mass $1 M_{\odot}$. 
Table A.2. Double compact-star merger DTD for $Z=0.02$.

\begin{tabular}{|c|c|c|c|c|c|c|c|c|c|c|}
\hline $\begin{array}{l}\log t \\
(\mathrm{Gyr})\end{array}$ & $\begin{array}{c}\text { mod. } 2 / 4 \\
\text { NSNS }\end{array}$ & $\begin{array}{c}\text { mod. } 2 / 4 \\
\text { BHNS }\end{array}$ & $\begin{array}{l}\text { mod. } 5 \\
\text { NSNS }\end{array}$ & $\begin{array}{l}\text { mod. } 5 \\
\text { BHNS }\end{array}$ & $\begin{array}{l}\text { mod. } 7 \\
\text { NSNS }\end{array}$ & $\begin{array}{l}\text { mod. } 7 \\
\text { BHNS }\end{array}$ & $\begin{array}{l}\text { mod. } 12 \\
\text { NSNS }\end{array}$ & $\begin{array}{l}\text { mod. } 12 \\
\text { BHNS }\end{array}$ & $\begin{array}{l}\text { mod. } 13 \\
\text { NSNS }\end{array}$ & $\begin{array}{c}\text { mod. } 13 \\
\text { BHNS }\end{array}$ \\
\hline-1.85 & & -9.715 & & -9.715 & & -7.751 & & & & -9.857 \\
\hline-1.75 & & -8.789 & & -8.790 & & -7.794 & & & & -8.928 \\
\hline-1.65 & & -8.618 & & -8.641 & & -7.818 & & -9.813 & & -8.757 \\
\hline-1.55 & -9.285 & -8.444 & -9.282 & -8.461 & -7.801 & -8.110 & -9.285 & -9.655 & -9.593 & -8.570 \\
\hline-1.45 & -9.667 & -8.723 & -9.699 & -8.757 & -7.881 & -8.278 & -9.667 & -9.588 & -9.975 & -8.884 \\
\hline-1.35 & -9.272 & -9.069 & -9.272 & -9.127 & -8.034 & -8.393 & -9.272 & -10.296 & -9.563 & -9.231 \\
\hline-1.25 & -8.754 & -9.164 & -8.752 & -9.267 & -7.967 & -8.632 & -8.754 & -11.010 & -9.027 & -9.345 \\
\hline-1.15 & -6.681 & -9.235 & -6.681 & -9.332 & -6.603 & -8.677 & -6.681 & -10.508 & -6.810 & -9.431 \\
\hline-1.05 & -6.170 & -9.392 & -6.170 & -9.419 & -6.320 & -8.664 & -6.170 & -10.378 & -6.297 & -9.599 \\
\hline-0.95 & -6.204 & -9.422 & -6.204 & -9.585 & -6.577 & -8.725 & -6.204 & -10.568 & -6.346 & -9.633 \\
\hline-0.85 & -6.368 & -9.459 & -6.368 & -9.720 & -6.848 & -8.847 & -6.368 & -10.876 & -6.505 & -9.674 \\
\hline-0.75 & -6.495 & -9.334 & -6.495 & -9.585 & -6.938 & -8.844 & -6.495 & -10.734 & -6.642 & -9.546 \\
\hline-0.65 & -6.574 & -9.063 & -6.574 & -9.532 & -7.012 & -8.869 & -6.574 & -10.918 & -6.713 & -9.280 \\
\hline-0.55 & -6.708 & -9.282 & -6.709 & -9.555 & -7.070 & -8.740 & -6.708 & -10.566 & -6.856 & -9.497 \\
\hline-0.45 & -6.795 & -9.226 & -6.795 & -9.520 & -7.151 & -8.841 & -6.795 & -10.245 & -6.943 & -9.436 \\
\hline-0.35 & -6.851 & -9.211 & -6.851 & -9.459 & -7.211 & -9.011 & -6.851 & -10.081 & -6.994 & -9.413 \\
\hline-0.25 & -6.900 & -9.383 & -6.900 & -9.520 & -7.268 & -8.973 & -6.900 & -9.782 & -7.047 & -9.577 \\
\hline-0.15 & -7.048 & -9.359 & -7.048 & -9.500 & -7.348 & -8.893 & -7.048 & -9.566 & -7.187 & -9.546 \\
\hline-0.05 & -7.162 & -9.191 & -7.162 & -9.357 & -7.463 & -8.845 & -7.162 & -9.218 & -7.305 & -9.378 \\
\hline 0.05 & -7.360 & -9.191 & -7.360 & -9.353 & -7.544 & -8.846 & -7.360 & -8.725 & -7.498 & -9.379 \\
\hline 0.15 & -7.505 & -9.082 & -7.504 & -9.297 & -7.665 & -8.738 & -7.505 & -8.472 & -7.642 & -9.270 \\
\hline 0.25 & -7.697 & -8.909 & -7.697 & -9.088 & -7.716 & -8.716 & -7.697 & -8.293 & -7.838 & -9.103 \\
\hline 0.35 & -8.115 & -9.034 & -8.115 & -9.310 & -7.878 & -8.639 & -8.115 & -8.198 & -8.224 & -9.219 \\
\hline 0.45 & -8.456 & -9.016 & -8.455 & -9.328 & -8.098 & -8.628 & -8.456 & 164 & -8.581 & -9.203 \\
\hline 0.55 & -8.258 & -8.950 & -8.258 & -9.429 & -8.308 & -8.640 & -8.258 & -8.155 & -8.415 & -9.151 \\
\hline 0.65 & -8.874 & -9.065 & -8.874 & -9.497 & -8.471 & -8.650 & -8.874 & -8.180 & -9.032 & -9.261 \\
\hline 0.75 & -8.659 & -9.212 & -8.659 & -9.551 & -8.692 & -8.611 & -8.659 & -8.243 & -8.839 & -9.406 \\
\hline 0.85 & -8.748 & -9.440 & -8.748 & -9.657 & -9.043 & -8.567 & -8.748 & -8.293 & -8.915 & -9.629 \\
\hline 0.95 & -9.321 & -9.515 & -9.321 & -9.601 & -8.855 & -8.494 & -9.321 & -8.413 & -9.327 & -9.704 \\
\hline 1.05 & -8.307 & -9.624 & -8.307 & -9.735 & -8.512 & -8.363 & -8.307 & -8.577 & -8.460 & -9.819 \\
\hline 1.15 & -8.021 & -9.637 & -8.021 & -9.669 & -8.164 & -8.316 & -8.021 & -8.633 & -8.169 & -9.827 \\
\hline 1.25 & -7.951 & -9.609 & -7.951 & -9.626 & -7.971 & -8.212 & -7.951 & -8.729 & -8.087 & -9.799 \\
\hline
\end{tabular}

Notes. Values are logarithmic in number of events per time bin after a starburst with total mass $1 M_{\odot}$. 
N. Mennekens and D. Vanbeveren: DTD of massive double compact star mergers $(R N)$

Table A.3. Double compact-star merger $r$-process element yields DTD for $Z=0.002$.

\begin{tabular}{|c|c|c|c|c|c|c|c|c|c|c|}
\hline $\begin{array}{l}\log t \\
(\mathrm{Gyr})\end{array}$ & $\begin{array}{c}\text { mod. } 2 / 4 \\
\text { NSNS }\end{array}$ & $\begin{array}{c}\text { mod. 2/4 } \\
\text { BHNS }\end{array}$ & $\begin{array}{l}\text { mod. } 5 \\
\text { NSNS }\end{array}$ & $\begin{array}{l}\text { mod. } 5 \\
\text { BHNS }\end{array}$ & $\begin{array}{l}\text { mod. } 7 \\
\text { NSNS }\end{array}$ & $\begin{array}{l}\text { mod. } 7 \\
\text { BHNS }\end{array}$ & $\begin{array}{c}\text { mod. } 12 \\
\text { NSNS }\end{array}$ & $\begin{array}{c}\text { mod. } 12 \\
\text { BHNS }\end{array}$ & $\begin{array}{c}\text { mod. } 13 \\
\text { NSNS }\end{array}$ & $\begin{array}{c}\text { mod. } 13 \\
\text { BHNS }\end{array}$ \\
\hline-2.05 & & -9.634 & & -9.515 & & -8.590 & & & & -9.776 \\
\hline-1.95 & & -8.359 & & -8.316 & & -8.632 & & & & -8.500 \\
\hline-1.85 & -10.899 & -8.030 & -10.338 & -7.969 & -10.847 & -8.469 & -10.899 & -10.506 & -11.194 & -8.171 \\
\hline-1.75 & -11.110 & -7.965 & -11.291 & -7.876 & -10.927 & -8.645 & -11.110 & -11.618 & -11.409 & -8.106 \\
\hline-1.65 & -11.275 & -8.236 & -11.785 & -8.205 & -10.627 & -8.364 & -11.275 & -11.176 & -11.542 & -8.379 \\
\hline-1.55 & -11.443 & -8.560 & -10.473 & -8.533 & -9.910 & -8.403 & -11.443 & -10.236 & -11.727 & -8.721 \\
\hline-1.45 & -11.779 & -8.792 & -10.926 & -8.766 & -9.334 & -8.354 & -11.779 & -10.450 & -12.055 & -8.967 \\
\hline-1.35 & -9.791 & -8.961 & -9.746 & -8.923 & -9.106 & -8.484 & -9.791 & -10.530 & -9.866 & -9.146 \\
\hline-1.25 & -10.419 & -9.096 & -10.430 & -9.055 & -9.162 & -8.314 & -10.419 & -10.642 & -10.674 & -9.282 \\
\hline-1.15 & -9.854 & -9.148 & -9.865 & -9.096 & -8.171 & -8.235 & -9.854 & -10.760 & -9.994 & -9.327 \\
\hline-1.05 & -9.304 & -9.066 & -9.274 & -8.997 & -7.968 & -8.272 & -9.304 & -11.214 & -9.419 & -9.233 \\
\hline-0.95 & -9.270 & -8.904 & -9.248 & -8.828 & -8.225 & -8.269 & -9.270 & -11.099 & -9.385 & -9.060 \\
\hline-0.85 & -9.190 & -8.737 & -9.170 & -8.661 & -8.514 & -8.262 & -9.190 & -11.010 & -9.317 & -8.886 \\
\hline-0.75 & -9.401 & -8.660 & -9.286 & -8.593 & -8.675 & -8.237 & -9.401 & -11.244 & -9.535 & -8.804 \\
\hline-0.65 & -9.556 & -8.653 & -9.539 & -8.591 & -8.841 & -8.222 & -9.556 & -11.806 & -9.688 & -8.789 \\
\hline-0.55 & -9.825 & -8.680 & -9.900 & -8.623 & -8.977 & -8.192 & -9.825 & -11.888 & -9.940 & -8.804 \\
\hline-0.45 & -10.113 & -8.715 & -10.047 & -8.663 & -9.184 & -8.159 & -10.113 & -12.397 & -10.217 & -8.827 \\
\hline-0.35 & -10.186 & -8.751 & -10.867 & -8.700 & -9.414 & -8.134 & -10.186 & -11.309 & -10.317 & -8.848 \\
\hline-0.25 & -10.625 & -8.719 & -11.673 & -8.720 & -9.624 & -8.080 & -10.625 & -9.502 & -10.757 & -8.800 \\
\hline-0.15 & -11.049 & -8.775 & & -8.729 & -9.925 & -8.047 & -11.049 & -10.340 & -11.188 & -8.850 \\
\hline-0.05 & & -8.750 & & -8.737 & -10.162 & -7.973 & & -10.028 & & -8.811 \\
\hline 0.05 & & -8.636 & & -8.721 & -10.421 & -7.968 & & -9.761 & & -8.686 \\
\hline 0.15 & & -8.379 & & -8.706 & -10.900 & -7.859 & & -9.383 & & -8.411 \\
\hline 0.25 & & -8.683 & & -8.687 & -11.074 & -7.809 & & -8.773 & & -8.732 \\
\hline 0.35 & -13.352 & -8.388 & & -8.670 & -11.266 & -7.932 & -13.352 & -8.845 & -13.636 & -8.436 \\
\hline 0.45 & -11.545 & -8.529 & -11.567 & -8.658 & -10.600 & -7.826 & -11.545 & -8.827 & -11.814 & -8.576 \\
\hline 0.55 & -11.276 & -8.676 & -10.927 & -8.640 & -10.242 & -7.778 & -11.276 & -8.591 & -11.541 & -8.729 \\
\hline 0.65 & -11.323 & -8.870 & -10.834 & -8.615 & -9.953 & -7.930 & -11.323 & -8.794 & -11.572 & -8.912 \\
\hline 0.75 & -11.349 & -8.918 & -11.026 & -8.619 & -9.377 & -7.856 & -11.349 & -8.740 & -11.573 & -8.953 \\
\hline 0.85 & -11.625 & -8.944 & -11.321 & -8.639 & -9.255 & -7.946 & -11.625 & -8.863 & -11.893 & -8.977 \\
\hline 0.95 & -11.436 & -8.985 & -11.271 & -8.683 & -9.217 & -7.903 & -11.436 & -8.832 & -11.671 & -9.017 \\
\hline 1.05 & & -9.067 & -11.675 & -8.747 & -9.221 & -8.077 & & -8.822 & & -9.100 \\
\hline 1.15 & & -9.171 & -12.231 & -8.805 & -9.186 & -8.068 & & -8.685 & & -9.205 \\
\hline 1.25 & & -9.318 & -12.867 & -8.887 & -9.246 & -8.019 & & -8.758 & & -9.351 \\
\hline
\end{tabular}

Notes. Values are logarithmic in $M_{\odot}$ per time bin after a starburst with total mass $1 M_{\odot}$. 
A\&A 589, A64 (2016)

Table A.4. Double compact-star merger $r$-process element yields for DTD for $Z=0.002$.

\begin{tabular}{|c|c|c|c|c|c|c|c|c|c|c|}
\hline $\begin{array}{l}\log t \\
\text { (Gyr) }\end{array}$ & $\begin{array}{c}\text { mod. } 2 / 4 \\
\text { NSNS }\end{array}$ & $\begin{array}{c}\text { mod. } 2 / 4 \\
\text { BHNS }\end{array}$ & $\begin{array}{l}\text { mod. } 5 \\
\text { NSNS }\end{array}$ & $\begin{array}{l}\text { mod. } 5 \\
\text { BHNS }\end{array}$ & $\begin{array}{l}\text { mod. } 7 \\
\text { NSNS }\end{array}$ & $\begin{array}{l}\text { mod. } 7 \\
\text { BHNS }\end{array}$ & $\begin{array}{c}\text { mod. } 12 \\
\text { NSNS }\end{array}$ & $\begin{array}{c}\text { mod. } 12 \\
\text { BHNS }\end{array}$ & $\begin{array}{c}\text { mod. } 13 \\
\text { NSNS }\end{array}$ & $\begin{array}{c}\text { mod. } 13 \\
\text { BHNS }\end{array}$ \\
\hline-1.85 & & -11.235 & & -11.235 & & -9.275 & & & & -11.381 \\
\hline-1.75 & & -10.316 & & 10.317 & & -9.314 & & & & -10.456 \\
\hline-1.65 & & -10.164 & & -10.204 & & -9.339 & & -11.375 & & -10.308 \\
\hline-1.55 & -11.156 & -10.082 & -11.153 & -10.116 & -9.710 & -9.671 & -11.156 & -11.216 & -11.464 & -10.219 \\
\hline-1.45 & & & & & & & & & & \\
\hline-1.3 & & & & & & & & & & \\
\hline-1.2 & & & & & & & & & & -10.913 \\
\hline & & & & & & & & & & -10.977 \\
\hline-1.05 & & & & & & & & & & -11.155 \\
\hline-0.95 & & & & & & & & & & -11.113 \\
\hline-0.85 & & & & & & & & & & -11.115 \\
\hline-0.75 & & & & & & & & & & \\
\hline-0.65 & & & & & & & & & & -10.674 \\
\hline & & & & & & & & & & \\
\hline & & & & & & & & & & \\
\hline & & & & & & & & & & \\
\hline-0.25 & & & & & & & & & & \\
\hline-0.15 & & & & & & & & & & \\
\hline-0.05 & & & & & & & & & & \\
\hline 0.05 & & & & & & & & & & -10.842 \\
\hline 0.15 & & & & & & & & & & -10.721 \\
\hline 0.25 & -9.645 & & & & & & & & & \\
\hline 0.35 & & & & & & & & & & \\
\hline & & & & & & & & & & \\
\hline & & & & & & & & & & \\
\hline 0.65 & -10.828 & & & & & & & & & -10.640 \\
\hline 0.75 & & & & & & & & & & -10.806 \\
\hline 0.85 & -10.703 & & & & -10.9 & & & & & -11.056 \\
\hline 0.95 & -11.254 & .969 & & -11.090 & -10.7 & -9 . & & -9. & & -11.159 \\
\hline 1.05 & -10.244 & & & & & & & & & -11.274 \\
\hline 1.15 & & & & & & & & & & -11.314 \\
\hline 1.25 & -9.887 & -11.113 & -9.887 & -11.136 & -9.911 & -9.497 & -9.887 & -10.281 & -10.021 & -11.301 \\
\hline
\end{tabular}

Notes. Values are logarithmic in $M_{\odot}$ per time bin after a starburst with total mass $1 M_{\odot}$.

Table A.5. Different models whose results are given in the present paper (rejected ones in italics).

\begin{tabular}{ccccccccc}
\hline \hline Model & ECSN & $\beta$ & $\alpha_{\mathrm{CE}}$ & $\begin{array}{c}\text { Fallback } \\
\text { model }\end{array}$ & $\begin{array}{c}\text { Period } \\
\text { distribution }\end{array}$ & LBV & $\begin{array}{c}\text { BH } \\
\text { kicks }\end{array}$ & $\begin{array}{c}\text { avg. NS } \\
\text { kick }\left(\mathrm{km} \mathrm{s}^{-1}\right)\end{array}$ \\
\hline $2 / 4$ & On & 1 & 0.5 & Rapid & Flat & On & On & 450 \\
5 & On & 1 & 0.5 & Delayed & Flat & On & On & 450 \\
6 & On & 1 & 1 & Rapid & Flat & On & On & 450 \\
7 & Off & 1 & 1 & Rapid & Flat & On & On & 450 \\
12 & On & 1 & 0.5 & Rapid & Flat & Off & On & 450 \\
13 & On & 1 & 0.5 & Rapid & Alternative & On & On & 450 \\
19 & On & 1 & 5 & Rapid & Flat & On & On & 450 \\
22 & On & 0.5 & 1 & Rapid & Flat & On & On & 450 \\
\hline
\end{tabular}

Notes. See text for the definition of parameters and their values, and see Mennekens \& Vanbeveren (2014) for details about these and other rejected models. 